Corresponding author address: Department of Biochemistry and Molecular Biology, University of Oklahoma Health Sciences Center, 940 Stanton L. Young Blvd., Oklahoma City, OK 73104, U.S.A., hiroyuki-matsumoto@ ouhsc.edu.

This manuscript is submitted as a contribution to the special issue dedicated to the 16th international Conference on Retinal Proteins held in Nagahama, Japan, in October 5-10, 2014 


\section{Abstract}

Rhodopsin regenerates through a non-covalent 11-cis retinal-opsin complex followed by a covalent formation of retinylidene Schiff base, rendering the $\beta$-ionone ring binding pocket a distinct physiological role.

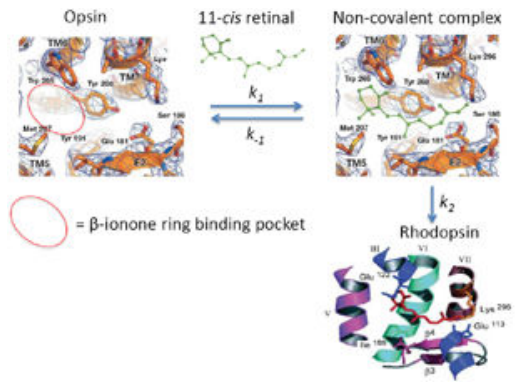

$k_{2}$ is likely to be the rate limiting step of rhodopsin regeneration because of slow Schiff base formation. The figures are modified from Scheerer et al. (2008) and from Imai et al. (2005).

\section{Background}

Dark adaptation assessed by recovery of visual threshold in humans follows biphasic kinetics for which two distinct classes of retinal proteins, iodopsin in cone cells and rhodopsin in rod cells, are responsible[3]. Both rhodopsin and iodopsin are prototypical retinal proteins consisting of 11-cis retinal (11-cis-R) and apoprotein moiety opsin, which are covalently linked through Schiff base between 11-cis- $\mathrm{R}$ and the $\varepsilon-\mathrm{NH}_{2}$ group of opsin polypeptide. In order for these visual pigments to function as a photon sensor, the once hydrolyzed Schiff base during visual excitation needs to be reestablished through a process called regeneration, a re-synthesis of the Schiff base. It is likely that the biphasic recovery of visual threshold reported by Hecht et al. [3] reflects the kinetic parameters of the two distinct visual pigments during regeneration. These opsins are encoded by distinct genes[4, 5] and, therefore, their expression must be responsible for the difference in the recovery of visual threshold.

Rhodopsin consists of 11-cis-R attached to the $\varepsilon$-amino group of lysine in opsin[6]. The regeneration of visual pigments, both rhodopsin and iodopsin, is a biochemical process in which Schiff base forms between the $\varepsilon$-amino group of lysine in opsin and the $\mathrm{C} 15$ aldehyde group of 11-cis-R. Since retinal has 4 carbon-carbon double bonds in the polyene side chain, there are 16 potentially existing isomers among which all-trans, 11-cis, and 9-cis isomers were established when George Wald's group initially characterized them and their binding to opsin[7]. Based on the fact that certain groups of retinal isomers and analogs form pigments with opsin, while others do not, Matsumoto and Yoshizawa[1] conjectured that opsin recognizes the longitudinal dimension of the ligand and predicted the existence of a $\beta$ ionone ring binding site in an hydrophobic pocket in opsin, and proposed a rhodopsin regeneration mechanism occurring in two discrete steps:

Step 1: Fast non-covalent binding of 11 -cis-R to the $\beta$-ionone ring-binding pocket 
Step 2: Slow formation of Schiff base between the retinal -CHO and the $\varepsilon$-amino group of the lysine

$$
\text { Opsin } \cdot 11 \text { cisR noncovalent complex } \rightarrow \text { rhodopsin }
$$

\begin{abstract}
Almost a quarter of a century later the existence of the $\beta$-ionone ring-binding site in a hydrophobic pocket in rhodopsin has been confirmed by structural studies $[8,9]$. Furthermore, the occupation of the $\beta$-ionone binding pocket with a proper ligand including all-trans-R was shown to activate visual transduction[10, 11], implying the physiological role of the $\beta$-ionone binding pocket in visual excitation.

In the past decades, it was well established that retinal proteins including visual pigments rhodopsin and iodopsin represent a prototypical protein family that led to an evolutional development of GPCR family proteins that evoke cellular signaling essential in the maintenance of life[12]. In contrast to visual pigments in which an inverse agonist ligand always occupies the binding site in a resting state, GPCRs dynamically bind their ligands to initiate cellular signals. In this regard the visual pigment regeneration is a step equivalent to that of ligand-binding step in other GPCR proteins. Therefore, comprehensive understanding of the regeneration mechanism of visual pigments would assist us to elucidate the activation of GPCRs by various ligands in detail.
\end{abstract}

\title{
2. Hypothesis and prediction
}

\section{The non-covalent complex formation between 11-cis-R and opsin accelerates the following Schiff base formation in rhodopsin regeneration}

Our hypothesis is based on the fact that opsin forms a visual pigment with only a subgroup of 16 isomers of retinal with a certain range of longitudinal lengths[13], suggesting that the non-covalent complex formation shown in Equation (1) restricts the Schiff base formation so that it proceeds only if the proximity and the orientation of the atomic groups involved in the Schiff base formation are optimized.

Assuming fixed bond angles and bond distances based on known crystallographic data of retinal, we evaluated the longitudinal lengths of 16 potential isomers of retinal (Fig. 1).

Although this is a rough estimation, the relative ratios of the longitudinal lengths between each isomer appear to be well represented. In Fig. 1A, all 16 isomers are drawn on a hexagonal grid and known retinal isomers that form pigments are indicated. In Fig. 1B, all the mono-cis and all-trans isomers are drawn overlapped, illustrating that the longitudinal lengths of 7-cis, 9-cis, and 11-cis, but not those of 13-cis and all-trans, would fall in a proper range, enabling the following Schiff base formation. An erroneous prediction that the 7,9dicis isomers may not form a pigment because it belongs to the 13-cis isomeric group[13], but in fact it does[14], has been interpreted based on the crystal structure of methyl 7,9-dicis 
retinoate because the longitudinal length of 7,9-dicis isomer in the crystal is shortened by the twisted polyene chain at carbons $\mathrm{C} 7-\mathrm{C} 10[15]$.

With availability of structural information of rhodopsin and its intermediates the first approximation on the longitudinal length of retinal chromophore in various configurations can now be evaluated based on structural data. Thus we calculated the distance between C6 from which the polyene chain extends to $\mathrm{C} 15$ at which the retinylidene Schiff base is formed with the $\varepsilon$-amino group of lysine based on PDB data; 1U19 (Rhodopsin)[16], 2PED (Isorhodopsin)[17], 2G87 (Bathorhodopsin)[18], and 3PX0 (Metarhodopsin II)[19], which is shown in Table 1.

The results in Table 1 support our early conjecture[13] that the longitudinal restriction of retinal binding site of rhodopsin determines the potency of retinal isomers to form pigments, or "figments" as Allen Kropf designated at the 1st Gordon Research Conference on "Physico-Chemical Aspects of the Visual Transduction Process" held in New Hampshire in 1978. Namely, the C6-C15 distances in rhodopsin and isorhodopsin are $10.0 \AA$ and $9.9 \AA$, respectively. The longitudinal polyene chain dynamically expands to $10.3 \AA$ and $11.0 \AA$ in bathorhodopsin and metarhodopsin II, respectively, after excitation is triggered. The reevaluation of the longitudinal length of retinal isomers based on their crystal structures strongly support our early assumption that rhodopsin regeneration follows a transient noncovalent formation of opsin-11-cis-R non-covalent complex between its cyclohexene ring and a hydrophobic cavity of opsin.

\section{Assay system of rhodopsin regeneration}

We assumed that rhodopsin regeneration follows a transient non-covalent formation of opsin-11-cis-R complex between its cyclohexene ring and a hydrophobic cavity of opsin. In order to prove our hypothesis we measured the second order rate constants $\left(k_{2}\right)$ of rhodopsin regeneration from 11-cis-R and opsin and compared the results with a model retinylidene Schiff base formation. Bovine opsin was prepared by conventional protocol[20]. Because digitonin slows the rhodopsin regeneration at concentrations higher than its critical micelle concentration (ca $0.1 \%$ ), the opsin extract was diluted by a factor of 41 into the reaction buffer at each $\mathrm{pH}$ in order to reduce the digitonin concentration to $0.012 \%$. Reaction mixture (total volume, $2.06 \mathrm{~mL}$ ) contained $1 \mu \mathrm{M}$ opsin and $7 \mu \mathrm{M}$ 11-cis-R as final concentrations. The reaction was started by adding 11-cis-R dissolved in $10 \mu \mathrm{L}$ of ethanol. Ethanol at this concentration did not affect rhodopsin regeneration. After the addition of 11-cis-R, the absorption spectra were measured[21] (Fig. 2): Spectrum 1, after the completion of regeneration reaction. Spectrum 2, after the addition of $10 \mathrm{mM} \mathrm{NH}_{2} \mathrm{OH}$. Spectrum 3, after complete bleaching of rhodopsin by orange light. Note that the difference spectra between 1 and 2 represent the random Schiff base between 11-cis-R and unidentified amines.

The results shown in Fig. 2 indicate that rhodopsin regenerates nearly 100\% throughout the $\mathrm{pH}$ range examined ( $\mathrm{pH}$ 5-10). The results of the experiments illustrated in Fig. 2 are plotted in Fig. 3. The plot at $530 \mathrm{~nm}$ (closed circles) shows that rhodopsin regenerates $100 \%$ independent of the $\mathrm{pH}$ of the reaction mixture. However, if one plots the absorbance increase at $480 \mathrm{~nm}$ (which is close to the absorption maximum of rhodopsin $498 \mathrm{~nm}$ thereby 
representing rhodopsin formation well), the regeneration kinetics would not be observed properly because the Schiff base formation between 11-cis-R and unidentified amines contribute significantly at this wavelength. Note that absorption "a" represents the real amount of regenerated rhodopsin, whereas absorption "b" represents the contribution of the unidentified random Schiff base at $480 \mathrm{~nm}$. This is illustrated in Fig. 3 by plotting the absorbance ratio b/a, which shows that the random Schiff base at $480 \mathrm{~nm}$ interferes severely with the true kinetics of rhodopsin regeneration, whereas the absorbance measurement at $530 \mathrm{~nm}$ escapes errors.

\section{The second order rate constant $k_{2}$ of rhodopsin regeneration is independent of $\mathrm{pH}$}

Using the assay system described in Fig. 2, the second order rate constant, $k_{2}$, of rhodopsin regeneration was measured under different $\mathrm{pH}$ conditions. Combined representation of equations (1) and (2) can be justified because the intermediary formation of opsin-11-cis-R non-covalent complex represented by equation (1) is likely to occur significantly faster than the formation of rhodopsin represented by equation (2). Thus the overall rhodopsin regeneration can be analyzed by equation (3) shown below where $k_{2}$ is an apparent second order rate constant involving [opsin] and [11-cis-R].

$$
\text { Opsin+11cis R } \stackrel{k_{2}}{\longrightarrow} \text { Rhodopsin (3) }
$$

The observed $k_{2}$ values shown in Fig. 4 indicate that the retinal Schiff base formation in rhodopsin occurs at a constant rate independent of $\mathrm{pH}$ of the reaction mixture. The $\mathrm{pH}$ independence further suggests that water solvents in the reaction mixture do not have access to the milieu of the retinal-binding pocket of rhodopsin during the Schiff base formation. We suspected that, because of the complex formation between opsin and 11-cis-R, the following covalent formation of Schiff base is likely to be accelerated. Therefore, we compared the Schiff base formation in rhodopsin and that in solution.

\section{Second order rate constant $\boldsymbol{k}_{2}$ of a model retinylidene Schiff base in solution}

We measured Schiff base formation between all-trans-R and N-a-acetyl-L-lysine in a model system and compared that of rhodopsin regeneration[21, 22]. In Fig. 5 the spectra of alltrans retinal and its Schiff base formed with N-a-acetyl-L-lysine (Fig. 5A) and its formation kinetics (Fig. 5B) are shown. The experimental details are described in the figure caption. From these results we calculated the second order rate constant $k_{2}$ of a model retinylidene Schiff base in comparison to that corresponding to $k_{2}$ in rhodopsin regeneration: The mean and standard deviation were calculated to be $6100 \pm 300 \mathrm{~mol}^{-1} \mathrm{~s}^{-1}\left(25^{\circ} \mathrm{C}, \mathrm{pH} 5-10\right)$ with the confidence of $p<0.01$ calculated from Fig. 4):

A model retinylidene Schiff base; $k_{2}=5.7 \times 10^{-4} \mathrm{M}^{-1} \mathrm{~s}^{-1}\left(25^{\circ} \mathrm{C}\right.$ in $22 \%$ ethanol/water, pH8.2) and Rhodopsin; $k_{2}=6100 \mathrm{M}^{-1} \mathrm{~s}^{-1}\left(25^{\circ} \mathrm{C}\right.$ in $0.1 \%$ digitonin/water, over the range of $\mathrm{pH} 5-10)$. 
These results indicate that opsin catalyzes/accelerates the formation of retinylidene Schiff base in rhodopsin approximately $10^{7}$ times compared to that in solution. The acceleration of $k_{2}$ is likely due to the non-covalent complex formation, which enables a proper proximity and alignment of the aldehyde - $\mathrm{CHO}$ group of retinal to the $\varepsilon$-amino group of active lysine. Elaborated model experiments in which the formation of retinylidene Schiff base in various micelle systems were reported by Cooper et al.[23], the results of which illustrate a slow formation of model Schiff base consistent with our results.

\section{Discussion}

\section{Additional studies on the inhibitory action of cyclohexenyl analogs upon the regeneration reaction of rhodopsin in vitro}

Additional evidence to support the existence of $\beta$-ionone ring binding site in opsin had been added later by two other groups[24, 25], further supporting the original hypothesis by Matsumoto and Yoshizawa[1].

\section{Regeneration of rhodopsin and iodopsin in vitro and in vivo}

Regeneration of chicken iodopsin in vitro occurs more than 500 times faster than that of rhodopsin[26]. More recently, Shichida's group investigated why such a huge difference in regeneration rate exists in the context of the three dimensional structure of rhodopsin[27, 28]. According to their account the glutamic acid-122 in rhodopsin contributes to the deceleration of its regeneration reaction. Thus, if the polar glutamic acid residue at 122 were eliminated, rhodopsin regeneration would be accelerated to the level of that of iodopsin. This notion was supported by an experiment in which the glutamic acid-122 of chicken rhodopsin was mutated into glutamine (E122Q), resulting in significant acceleration of regeneration reaction. The suppression of $k_{2}$ in wild-type rhodopsin with glutamic acid-122 and the release of suppression, i.e., acceleration, by the E122Q mutation could be due to a diminished population of the opsin-11-cis-R non-covalent complex shown in equations (1) and (2). A further examination of the E122Q mutant rhodopsin would clarify the mechanism. In the in vitro experiments in which artificial pigments are studied, reasons why certain groups of retinal analogs and isomers can form a pigment, although at substantially slow rates and yields, should be reevaluated in the context that the pigment formation follows the two distinct steps. For example, a retinal analog lacking the methyl group at the C5 position forms a pigment at an approximately 100 times slower rate[29]. The reason why the removal of a methyl group from the $\beta$-ionone ring could be understood if the two distinct steps involved in rhodopsin regeneration are evaluated separately. Retinal analog studies suggest that as long as the $\beta$-ionone ring binding-site can accommodate the chemical structure and the formation of non-covalent retinal-opsin complex is formed in favor of the following Schiff base formation, a retinal analog can form a pigment; for example, openring analogs[30, 31] and $\mathrm{a}$-analogs[32].

In vivo, whether the difference of regeneration rates between iodopsin and rhodopsin is responsible for determining the visual threshold as observed by Hecht et al.[3] needs careful evaluation because the supply of 11-cis-R for regeneration differs between cones and rods in vivo. Both mechanisms, i.e., acceleration of regeneration controlled by the supply of 
available 11-cis-R and acceleration attributed to the intrinsic property of the retinal-binding site structure, are not mutually exclusive.

\section{Role of $\beta$-ionone ring-binding site in excitation process}

In contrast to vertebrate visual pigments, bacteriorhodopsin (bR) does not require occupancy of a cyclohexene ( $\beta$-ionone ring) binding site[33]. However, there is a clear preference of a 6-s-trans conformer over a 6-s-cis conformer in bacteriorhodopsin[34], suggesting that there is some ring recognition or constraint rather than a nonselective cavity. Nonetheless, bR regeneration is likely to start from binding of retinal into a crevice from the polyene chain ($\mathrm{CHO}$ ) side, not from the cyclohexene ( $\beta$-ionone) end[35]. The structural difference between visual pigments and bacteriorhodopsin, i.e., the former has a $\beta$-ionone ring-binding site, whereas the latter does not, could be reflected in the fast kinetic phases of both retinal proteins during photoisomerization. In order to explain molecular basis for fast kinetics of 11-cis to all-trans isomerization of rhodopsin, two models "bicycle-pedal model" [36] and "hula-twist model"[37] have been proposed. These models are based on the idea that the both ends, i.e., protonated Schiff base and the $\beta$-ionone ring of retinal chromophore, are tethered in the retinal-binding pocket. The restriction of the retinal chromophore in the hydrophobic pocket appears to enable highly efficient and sensitive photo-isomerization in rhodopsin. In fact, the 11-cis to all-trans isomerization of rhodopsin occurs at an extremely high speed, within $\sim 200$ femtoseconds, at room temperature[38]. In contrast, the photoisomerization in bacteriorhodopsin (in this case, all-trans to 13-cis conversion) takes place at a slightly slower rate in the range of 0.5 picoseconds, i.e., 500 femtoseconds[39]. We interpret the difference in the speed of conjugated polyene isomerization to arise from a lack or loose tethering of chromophore at the both ends in the case of bacteriorhodopsin. Logunov et al. [40] reported a 2.5-4 psec transition in absorption changes of protonated retinal Schiff base in 13-cis or all-trans conformation, which is consistent with the fluorescent transition data of protonated 11-cis retinal isomer in methanol reported by Kandori et al. [41] and represents the isomerization process in organic solvents. Thus the protein moiety of bacteriorhodopsin catalyzes the photoisomerization by the factor of at least 10 compared to that in solution. In the case of protonated 11-cis isomer Kandori et al. observed two fluorescent decay components [41]; the fast femtosecond (90-600 fsec) and the slow picosecond (2-3 ps) components. Kandori et al.[41] suggested that the fast femtosecond component is due to inhomogeneous distribution of the protonated retinal Schiff base in the ground state. However, Logunov et al.[40] argued that there are other possibilities for the interpretation of the fast femtosecond component. Nonetheless, these results are consistent with our idea that the tethering of retinal molecules in rhodopsins affects the physicochemical properties of retinal photoisomerization and catalyzes it toward acceleration.

Elegant works $[18,42]$ by Nakamichi and Okada in which difference electron density maps of crystals were collected between rhodopsin, bathorhodopsin and lumirhodopsin clearly snapshot the role of the $\beta$-ionone ring-binding pocket of rhodopsin in the early excitation process: In the transition from rhodopsin to bathorhodopsin the difference electron density maps imply that the both ends of the 11-cis-R are tightly tethered[18], apparently causing a hindrance within the bathorhodopsin chromophore, and this structural hindrance will 
eventually be relaxed thermally in the following lumirhodopsin stage by the departure of the cyclohexene ring from the original binding location in rhodopsin and bathorhodopsin[42].

The retinal-binding pocket consists of the $\beta$-ionone ring-binding pocket buried deep in the cavity surrounded by a bundle of hydrophobic a-helices and the molecular milieu near the retinylidene Schiff base. The molecular environment of the retinal-binding pocket exhibits significant variation between different visual pigment species and bacteriorhodopsin, rendering unique physicochemical properties such as pKa of the protonation of Schiff base and its dissociation constant. For example, while the retinylidene Schiff base of bovine rhodopsin is completely shielded from the titration[43], those of octopus rhodopsin (pKa $=10.4$ ) and Gecko cone pigment ( $\mathrm{pKa}=9.9$ ), can be titrated[44]. The retinylidene Schiff base in iodopsin is exposed to solvent milieu and its chromophore is exchangeable with 9-cis retinal added in the solution[45]. The dissociation of retinylidene Schiff base appears to be a general property of cone pigments because it was also reported in Gecko P521 pigment[46]. The dissociable nature of retinylidene Schiff base in iodopsin renders the accessibility to chloride ions added to the solvent in vitro[47]. The dissociable property of cone pigments caused by the different molecular milieu in the retinal-binding pocket may contribute to fundamental difference in response properties between rods and cones[48]: cones are 25- to 100-fold less sensitive to light than rods and their photoresponses are several fold faster in kinetics, which is attributed to such difference in the dissociable property of retinylidene Schiff base of cone pigments.

\section{Role of the $\beta$-ionone ring-binding site in the adaptation process; the relevance to visual threshold data}

Kefalov et al.[11] reported the differentiated roles of the non-covalent binding of 11-cisretinal to opsin in the dark between rods and cones of salamander. They conclude: "In rods, 11-cis-retinal produces a transient activation of the phototransduction cascade that precedes sensitivity recovery, thus slowing dark adaptation. In cones, 11-cis-retinal immediately deactivates phototransduction. Thus, the initial (non-covalent) binding of the same ligand to two very similar $\mathrm{G}$ protein receptors, the rod and cone opsins, activates one and deactivates the other, contributing to the remarkable difference in the rates of rod and cone dark adaptation." Whether or not the difference in the adaptive properties between rod pigment and cone pigment observed in salamander photoreceptors is applicable to all vertebrate retinae remains to be determined; for example, two distinct sets of parameters, for example, one that originates in the $\beta$-ionone ring-binding site structure and the other in the downstream transduction cascade, could affect the adaptation process. One such example has been reported[49]. Also, with the same retinal-binding pocket, a case in which a different retinal analog modified in the ring structure with 4-OH substitution affected the adaptation parameters was reported[50]. Nonetheless, the behaviors of salamander rod-cone system, if applicable to humans, would contribute significantly to quicker recovery of photopic (cone) vision threshold that Hecht et al.[3] reported in 1935. Future investigations should be based on structural data on overall retinal binding pocket consisting of the $\beta$ ionone ring-binding site buried deep and the molecular milieu surrounding the protonated retinylidene Schiff base. The differences of the molecular parameters among visual pigment species should result in distinct physiological responsiveness between rods and cones. 
In summary, the existence of a $\beta$-ionone ring binding site in a prototypical GPCR receptor rhodopsin that was predicted in 1975 has been confirmed by structural works reported early this century. Dark regeneration of rhodopsin from 11-cis-R and opsin is likely to involve a non-covalent complex. The existence of the non-covalent complex likely accelerates the Schiff base formation via the proximity and proper alignment of the retinal -CHO and the $\varepsilon$ amino group of lysine-296. Participation of the non-covalent complex in photoreceptor transduction is shown by the series of works by Kefalov et al.[10, 11]. Curiously, the noncovalent occupation of 11-cis $\mathrm{R}$ in the $\beta$-ionone ring-binding pocket exerts opposite effects on the photoreceptor transduction cascade, slowing the dark adaptation in rods while accelerating in cones. The acceleration of retinal Schiff base formation via the non-covalent 11-cis-R-opsin complex in vitro and the activation/inactivation of photoreceptor transduction cascade by the occupation of $\beta$-ionone ring binding site in the complex in situ illustrate a beautiful synergistic action in the dark adaptation that has been achieved in the long evolutionary history of vertebrate photoreceptor transduction. Moreover, the $\beta$-ionone ringbinding pocket appears to contribute in the excitation process by tethering the both ends of the elongated conjugated polyene side chain of retinal chromophore thereby enabling extremely rapid photoisomerization in vivo. As more structural data become available on retinoid proteins including visual pigments, inquiries on the role of retinal binding site in physicochemical events, biochemical reactions and their physiological manifestations could be understood thoroughly in the future.

\section{Acknowledgments}

This project was supported by grants from NIH EY017888, Oklahoma Center for Advancement of Science and Technology (OCAST) HR10-120 and OUHSC BMB Bridge grant. We thank Dr. Blaine Mooers for assisting with the use of PyMOL.

\section{References}

1. Matsumoto H, Yoshizawa T. Existence of a beta-ionone ring-binding site in the rhodopsin molecule. Nature. 1975; 258:523-6. [PubMed: 1196384]

2. Cordes EH, Jencks WP. On the Mechanism of Schiff Base Formation and Hydrolysis. J Amer Chem Soc. 1962; 84:832-7.

3. Hecht S, Haig C, Wald G. The Dark Adaptation of Retinal Fields of Different Size and Location. J Gen Physiol. 1935; 19:321-37. [PubMed: 19872930]

4. Nathans J, Thomas D, Hogness DS. Molecular genetics of human color vision: the genes encoding blue, green, and red pigments. Science. 1986; 232:193-202. [PubMed: 2937147]

5. Okano T, Kojima D, Fukada Y, Shichida Y, Yoshizawa T. Primary structures of chicken cone visual pigments: vertebrate rhodopsins have evolved out of cone visual pigments. Proc Natl Acad Sci U S A. 1992; 89:5932-6. [PubMed: 1385866]

6. Bownds D. Site of attachment of retinal in rhodopsin. Nature. 1967; 216:1178-81. [PubMed: 4294735]

7. Hubbard R, Wald G. Cis-trans isomers of vitamin A and retinene in the rhodopsin system. J Gen Physiol. 1952; 36:269-315. [PubMed: 13011282]

8. Palczewski K, Kumasaka T, Hori T, Behnke CA, Motoshima H, Fox BA, Le Trong I, Teller DC, Okada T, Stenkamp RE, et al. Crystal structure of rhodopsin: A G protein-coupled receptor. Science. 2000; 289:739-45. [PubMed: 10926528]

9. Li J, Edwards PC, Burghammer M, Villa C, Schertler GF. Structure of bovine rhodopsin in a trigonal crystal form. J Mol Biol. 2004; 343:1409-38. [PubMed: 15491621] 
10. Kefalov VJ, Carter Cornwall M, Crouch RK. Occupancy of the chromophore binding site of opsin activates visual transduction in rod photoreceptors. J Gen Physiol. 1999; 113:491-503. [PubMed: 10051522]

11. Kefalov VJ, Crouch RK, Cornwall MC. Role of noncovalent binding of 11-cis-retinal to opsin in dark adaptation of rod and cone photoreceptors. Neuron. 2001; 29:749-55. [PubMed: 11301033]

12. Audet M, Bouvier M. Restructuring G-protein- coupled receptor activation. Cell. 2012; 151:14-23. [PubMed: 23021212]

13. Matsumoto H, Yoshizawa T. Recognition of opsin to the longitudinal length of retinal isomers in the formation of rhodopsin. Vision Res. 1978; 18:607-9. [PubMed: 664347]

14. DeGrip WJ, Liu RS, Ramamurthy V, Asato A. Rhodopsin analogues from highly hindered 7-cis isomers of retinal. Nature. 1976; 262:416-8. [PubMed: 958397]

15. Matsumoto H, Liu RSH, Simmons CJ, Seff K. Longitudinal Restrictions of the Binding Site of Opsin As Measured with Retinal Isomers and Analogues. J Amer Chem Soc. 1980; 102:4259-62.

16. Okada T, Sugihara M, Bondar AN, Elstner M, Entel P, Buss V. The retinal conformation and its environment in rhodopsin in light of a new 2.2 A crystal structure. J Mol Biol. 2004; 342:571-83. [PubMed: 15327956]

17. Nakamichi H, Buss V, Okada T. Photoisomerization mechanism of rhodopsin and 9-cis-rhodopsin revealed by x-ray crystallography. Biophys J. 2007; 92:L106-8. [PubMed: 17449675]

18. Nakamichi H, Okada T. Crystallographic analysis of primary visual photochemistry. Angew Chem Int Ed Engl. 2006; 45:4270-3. [PubMed: 16586416]

19. Choe HW, Kim YJ, Park JH, Morizumi T, Pai EF, Krauss N, Hofmann KP, Scheerer P, Ernst OP. Crystal structure of metarhodopsin II. Nature. 2011; 471:651-5. [PubMed: 21389988]

20. Matsumoto H, Horiuchi K, Yoshizawa T. Effect of digitonin concentration on regeneration of cattle rhodopsin. Biochim Biophys Acta. 1978; 501:257-68. [PubMed: 563728]

21. Matsumoto H, Yoshizawa T. Rhodopsin regeneration is accelerated via noncovalent 11-cis retinalopsin complex--a role of retinal binding pocket of opsin. Photochem Photobiol. 2008; 84:985-9. [PubMed: 18399914]

22. Matsumoto, H. Kinetic studies of rhodopsin regeneration (in Japanese). Kyoto University; 1977.

23. Cooper A, Dixon SF, Nutley MA, Robb JL. Mechanism of retinal Schiff base formation and hydrolysis in relation to visual pigment photolysis and regeneration: resonance Raman spectroscopy of a tetrahedral carbinolamine intermediate and oxygen-18 labeling of retinal at the metarhodopsin stage in photoreceptor membranes. J Amer Chem Soc. 1987; 109:7254-63.

24. Daemen FJ. The chomophore binding space of opsin. Nature. 1978; 276:847-8. [PubMed: 723961]

25. Crouch RK, Veronee CD, Lacy ME. Inhibition of rhodopsin regeneration by cyclohexyl derivatives. Vision Res. 1982; 22:1451-56. [PubMed: 6222537]

26. Wald G, Brown PK, Smith PH. Iodopsin. J Gen Physiol. 1955; 38:623-81. [PubMed: 14367777]

27. Imai H, Kuwayama S, Onishi A, Morizumi T, Chisaka O, Shichida Y. Molecular properties of rod and cone visual pigments from purified chicken cone pigments to mouse rhodopsin in situ. Photochem Photobiol Sci. 2005; 4:667-74. [PubMed: 16121275]

28. Kuwayama S, Imai H, Morizumi T, Shichida Y. Amino acid residues responsible for the meta-III decay rates in rod and cone visual pigments. Biochemistry. 2005; 44:2208-15. [PubMed: 15697246]

29. Kropf A. Is proton transfer the initial photochemical process in vision? Nature. 1976; 264:92-4. [PubMed: 1004544]

30. Crouch R, Or YS. Opsin pigments formed with acyclic retinal analogues: Minimum 'ring portion'requirements for opsin pigment formation. FEBS Letters. 1983; 158:139-42.

31. Zhang B-W, Asato A, Denny M, Mirzadegan T, Trehan A, Liu R. Isomers, visual pigment, and bacteriorhodopsin analogs of 3, 7, 13-trimethyl-10-isopropyl-2, 4, 6, 8, 10-tetradecapentaenal and 3, 7, 11-trimethyl-10-isopropyl-2, 4, 6, 8, 10-dodecapentaenal (two ring open retinal analogs). Bioorganic Chemistry. 1989; 17:217-23.

32. Asato AE, Zhang B-W, Denny M, Mirzadegan T, Seff K, Liu RS. A study of the binding site requirements of rhodopsin using isomers of a-retinal and 5-substituted a-retinal analogs. Bioorganic Chemistry. 1989; 17:410-21. 
33. Towner P, Gaertner W, Walckhoff B, Oesterhelt D, Hopf H. Regeneration of rhodopsin and bacteriorhodopsin. The role of retinal analogues as inhibitors. Eur J Biochem. 1981; 117:353-9. [PubMed: 6456145]

34. Harbison GS, Smith SO, Pardoen JA, Courtin JM, Lugtenburg J, Herzfeld J, Mathies RA, Griffin RG. Solid-state carbon-13 NMR detection of a perturbed 6-s-trans chromophore in bacteriorhodopsin. Biochemistry. 1985; 24:6955-62. [PubMed: 4074732]

35. Isralewitz B, Izrailev S, Schulten K. Binding Pathway of Retinal to Bacterio-Opsin: A Prediction by Molecular Dynamics Simulations. Biophys J. 1997; 73:2972-9. [PubMed: 9414212]

36. Warshel A. Bicycle-pedal model for the first step in the vision process. Nature. 1976; 260:679-83. [PubMed: 1264239]

37. Liu RS, Asato AE. The primary process of vision and the structure of bathorhodopsin: a mechanism for photoisomerization of polyenes. Proc Natl Acad Sci U S A. 1985; 82:259-63. [PubMed: 3855551]

38. Schoenlein RW, Peteanu LA, Mathies RA, Shank CV. The first step in vision: femtosecond isomerization of rhodopsin. Science. 1991; 254:412-5. [PubMed: 1925597]

39. Herbst J, Heyne K, Diller R. Femtosecond infrared spectroscopy of bacteriorhodopsin chromophore isomerization. Science. 2002; 297:822-5. [PubMed: 12161649]

40. Logunov SL, Song L, El-Sayed MA. Excited-state dynamics of a protonated retinal Schiff base in solution. J Phys Chem. 1996; 100:18586-91.

41. Kandori H, Katsura Y, Ito M, Sasabe H. Femtosecond Fluorescence Study of the Rhodopsin Chromophore in Solution. J Am Chem Soc. 1995; 117:2669-70.

42. Nakamichi H, Okada T. Local peptide movement in the photoreaction intermediate of rhodopsin. Proc Natl Acad Sci U S A. 2006; 103:12729-34. [PubMed: 16908857]

43. Steinberg G, Ottolenghi M, Sheves M. pKa of the protonated Schiff base of bovine rhodopsin. A study with artificial pigments. Biophys J. 1993; 64:1499-1502. [PubMed: 8391868]

44. Liang J, Steinberg G, Livnah N, Sheves M, Ebrey TG, Tsuda M. The pKa of the protonated Schiff bases of gecko cone and octopus visual pigments. Biophys J. 1994; 67:848-54. [PubMed: 7948697]

45. Matsumoto H, Tokunaga F, Yoshizawa T. Accessibility of the iodopsin chromophore. Biochim Biophys Acta. 1975; 404:300-8. [PubMed: 1182163]

46. Crescitelli F. The gecko visual pigment: the dark exchange of chromophore. Vision Res. 1984; 24:1551-3. [PubMed: 6533985]

47. Shichida Y, Kato T, Sasayama S, Fukada Y, Yoshizawa T. Effects of chloride on chicken iodopsin and the chromophore transfer reactions from iodopsin to scotopsin and B-photopsin. Biochemistry. 1990; 29:5843-8. [PubMed: 2383562]

48. Kefalov VJ, Estevez ME, Kono M, Goletz PW, Crouch RK, Cornwall MC, Yau KW. Breaking the covalent bond--a pigment property that contributes to desensitization in cones. Neuron. 2005; 46:879-90. [PubMed: 15953417]

49. Isayama T, Chen Y, Kono M, Degrip W, Ma J-X, Crouch R, Makino C. Differences in the pharmacological activation of visual opsins. Visual neuroscience. 2006; 23:899-908. [PubMed: 17266782]

50. Corson D, Cornwall M, MacNichol E, Mani V, Crouch R. Transduction noise induced by 4hydroxy retinals in rod photoreceptors. Biophys J. 1990; 57:109. [PubMed: 2297557]

\section{Biography}

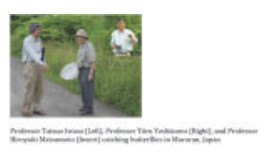

Professor Hiroyuki Matsumoto earned PhD in Biophysics with Professor T. Yoshizawa's at Kyoto University. Based on the knowledge on the binding capability of retinal isomers and 
analogs available at that time, he predicted the existence of $\beta$-ionone ring binding site in opsin. The prediction was verified by structural studies a quarter of a century later by multiple groups. The physiological roles of the $\beta$-ionone ring-binding pocket of opsin have been gradually revealed. He is Professor of Biochemistry and Molecular Biology at University of Oklahoma Health Sciences Center and Specially Appointed Professor at Kurume University School of Medicine.

Professor Tatsuo Iwasa earned PhD of Biophysics in Professor T. Yoshizawa's Laboratory in Kyoto University and studied early photoproducts of bacterorhodopsin. He assumed Assistant Professor position at Tohoku University and expanded the bacteriorhodopsin study using synthetic pigments with various retinal analogues. He also studied on halorhodopsin in Professor Dieter Oesterhelt's Laboratory at Max Planck Institute of Biochemistry as an Alexander von Humboldt Fellow. Recently his group isolated a new strain of Halorubrum species and found three retinal protein genes. He is Professor of Applied Sciences at Muroran Institute of Technology.

Professor Tôru Yoshizawa discovered early photoproducts of both vertebrate and invertebrate visual pigments. He expanded the knowledge to bacterial retinal proteins. The universal concept of early photoproducts in biological pigments has been further extended to other physiologically important biological pigments not only in the animal kingdom, but also in the plant kingdom such as in phytochrome. More recently, based on the primary structures of opsins, his group proposed that cone photoreceptors were originated and evolved into rod photoreceptors in the evolutionary process of vertebrate vision. Dr. Yoshizawa is Professor Emeritus at Kyoto University. 


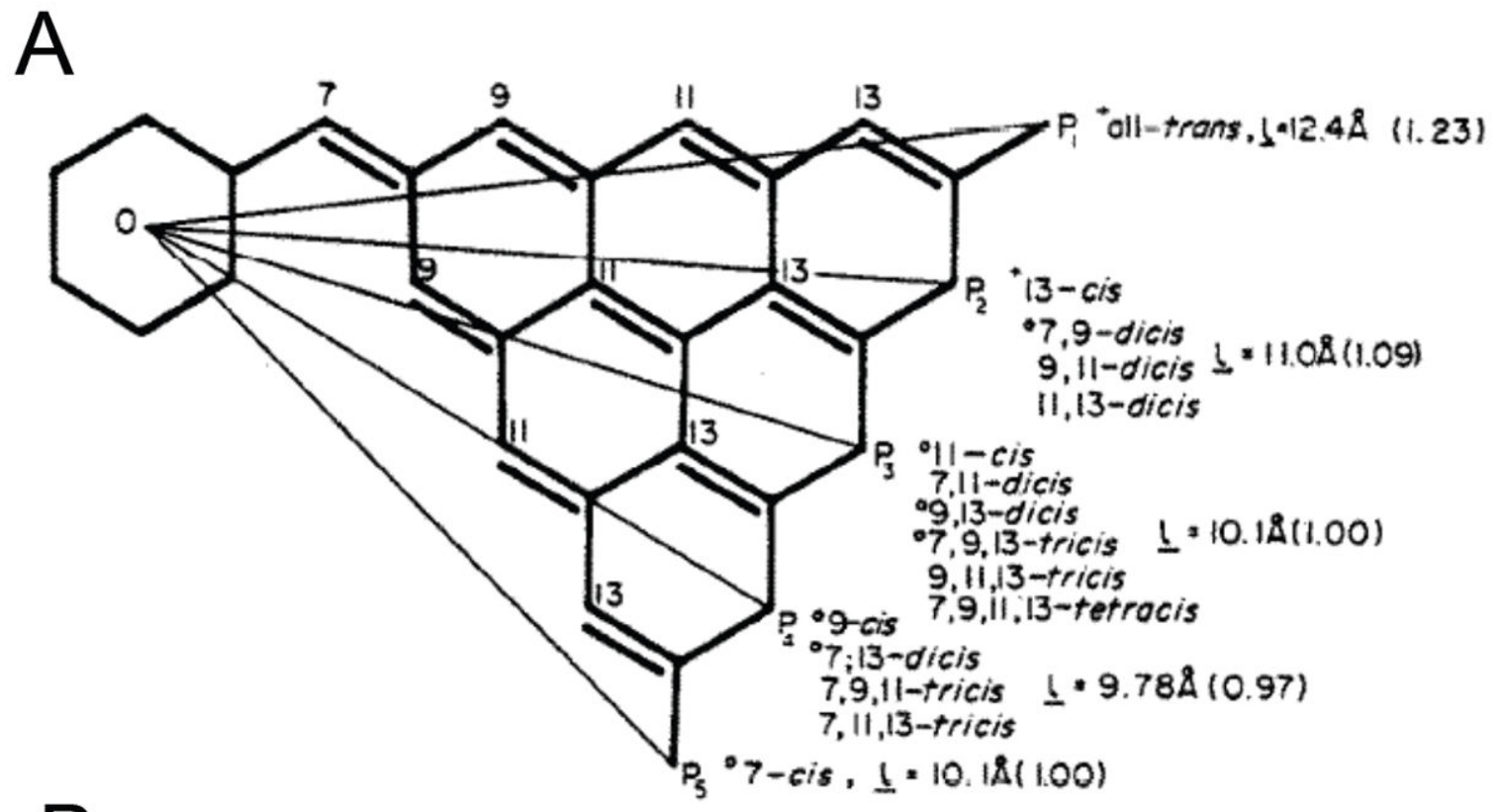

B

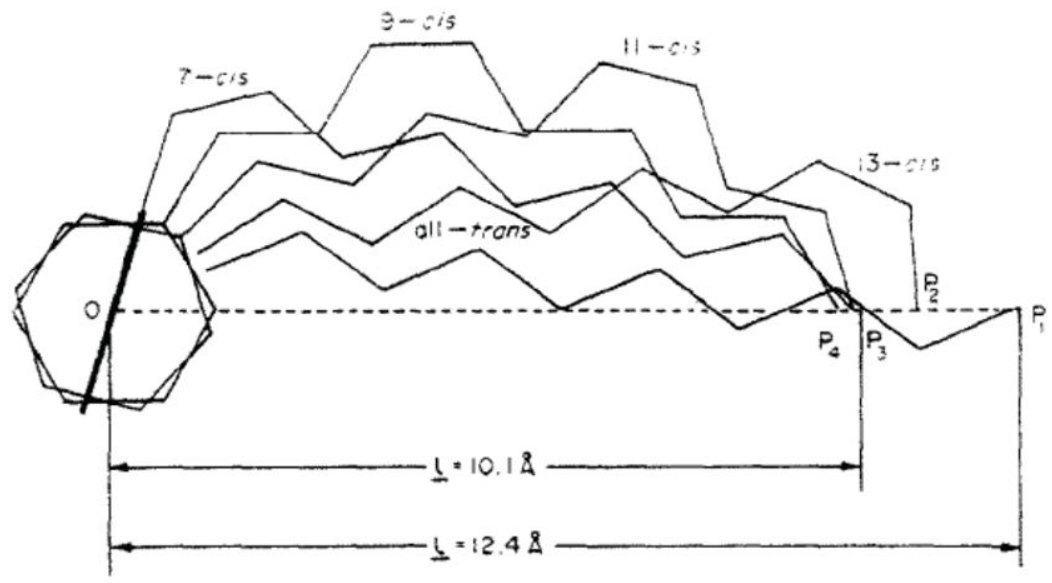

Fig. 1.

A. Active retinal isomers, which can form photosensitive pigments in combination with opsin, are marked with a superscript circle $\left(^{\circ}\right)$, and inactive ones with a cross $(+)$. Other isomers have never been examined. All the active retinal isomers except 7,9-dicis retinal can be classified into two groups, $10.1 \AA$ (11-cis and 7-cis groups) and $9.78 \AA$ (9-cis group) in terms of the longitudinal length $l$. Thus the reactive group has the same or a smaller value in $l$ compared to that of the active 11-cis group. In contrast, inactive isomers have much greater $l$ values: all-trans $(12.4 \AA)$ and 13-cis $(11.0 \AA)$ retinal. C15 of each isomer group is designated P1 (all-trans), P2 (13-cis), P3 (11-cis), P4 (9-cis), and P5 (7-cis), respectively.

The number in the parenthesis indicates the relative longitudinal length of each isomer group normalized to 11-cis retinal group. B. All the mono-cis and all-trans isomers are superimposed for comparison. Reproduced from reference 14 with the publisher's permission. 


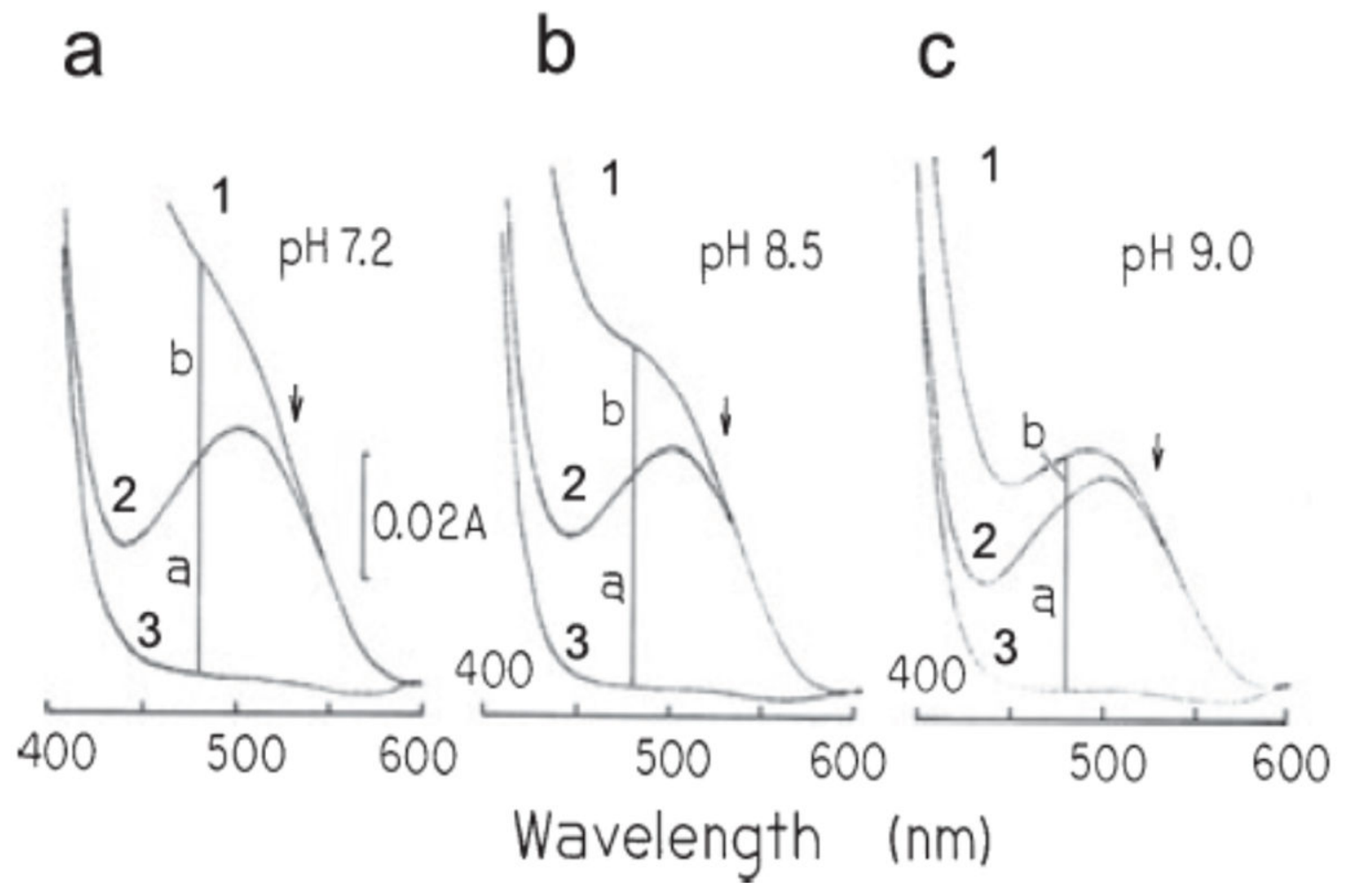

Fig. 2.

Rhodopsin regeneration under different $\mathrm{pH}$ conditions. The absorbance of both rhodopsin (designated "a") and a random Schiff base (designated "b") contribute to the absorbance at $480 \mathrm{~nm}$. Rhodopsin regeneration was monitored at $530 \mathrm{~nm}$ (shown by arrow) at which wavelength the contribution of a random Schiff base is negligible. Spectrum 1, after the completion of regeneration reaction. To achieve the completion we waited at least $60 \mathrm{~min}$ before we proceeded to the measurement of spectrum 2. Spectrum 2, after the addition of 10 $\mathrm{mM} \mathrm{NH} \mathrm{H}_{2} \mathrm{OH}$. Spectrum 3, after complete bleaching of rhodopsin by orange light. Reproduced from reference 22 with the publisher's permission. 


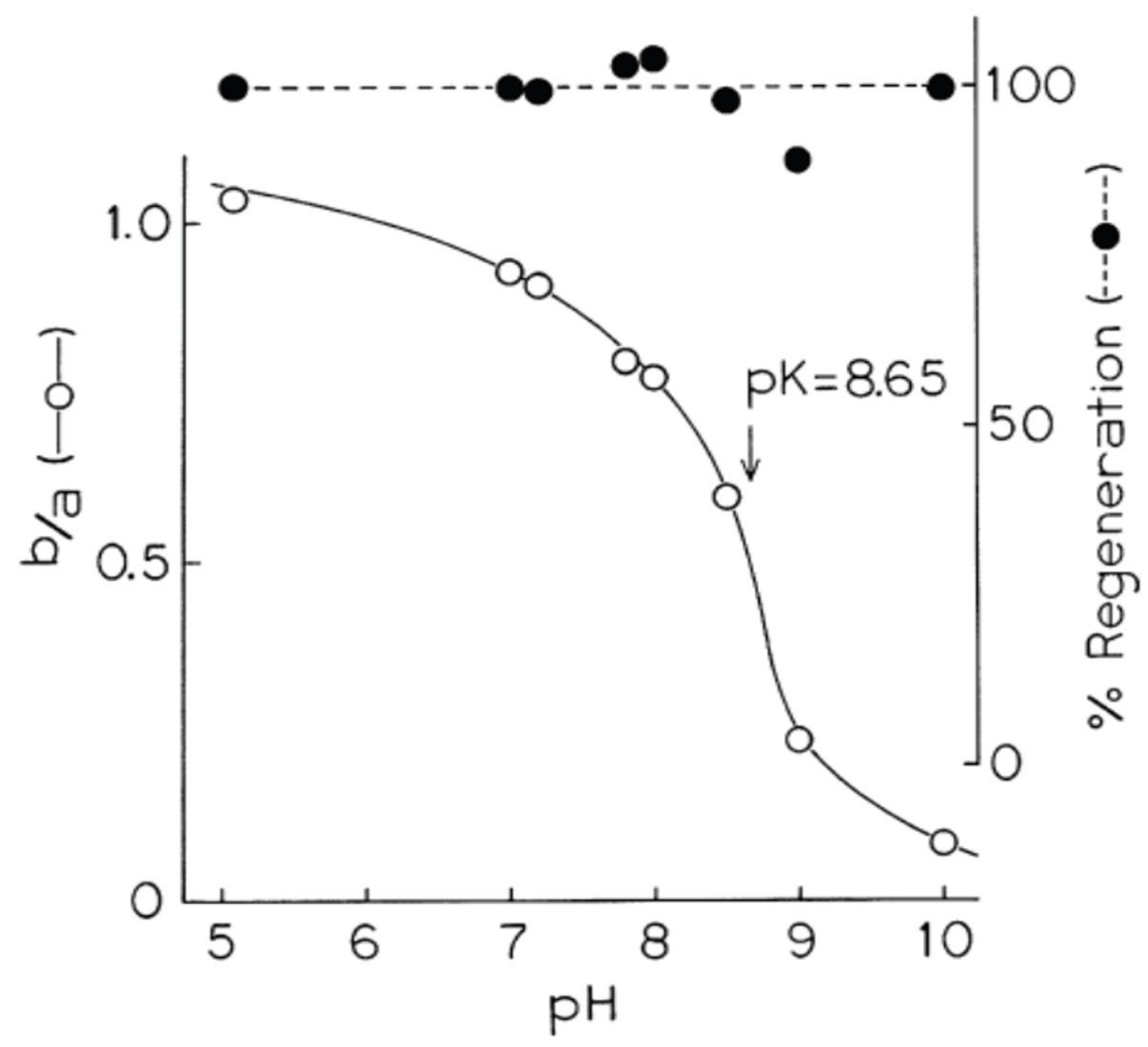

Fig. 3.

$\mathrm{pH}$ dependence of the formation of random Schiff base and the regenerated amount of rhodopsin. The ratio b/a indicates the contribution of the random Schiff base and rhodopsin, respectively at $480 \mathrm{~nm}$. Note that b/a diminishes to zero as $\mathrm{pH}$ increases to the alkaline region because the random Schiff base becomes deprotonated, the $\mathrm{pK}$ value of which was 8.65. Reproduced from reference 22 with the publisher's permission. 


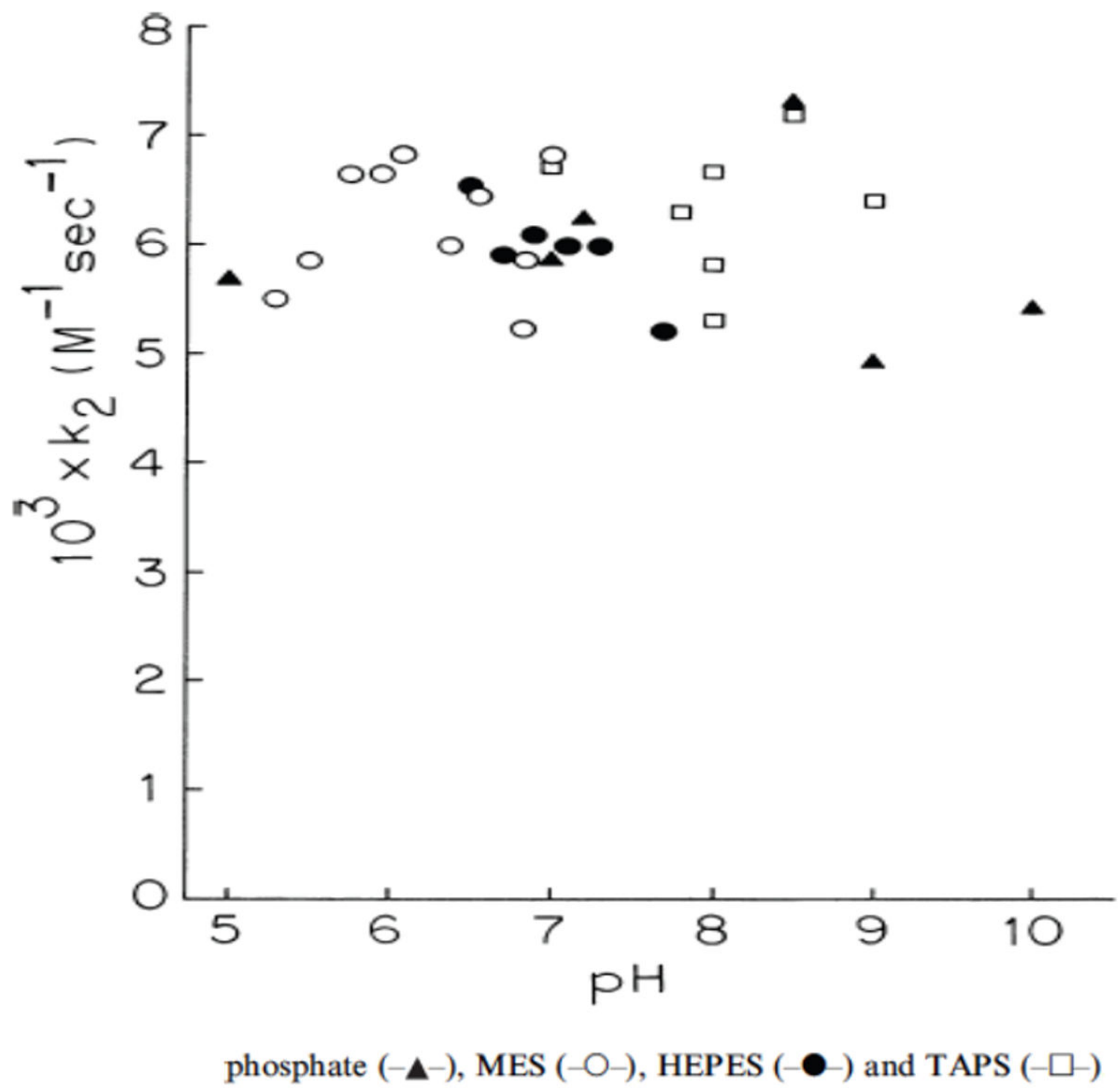

Fig. 4.

$k_{2}$ is independent of $\mathrm{pH}$. The mean and standard deviation are $6100 \pm 300 \mathrm{M}^{-1} \mathrm{~s}^{-1}\left(25^{\circ} \mathrm{C}, \mathrm{pH}\right.$ $5-10), \mathrm{p}<0.01$. Reproduced from reference 22 with the publisher's permission. 


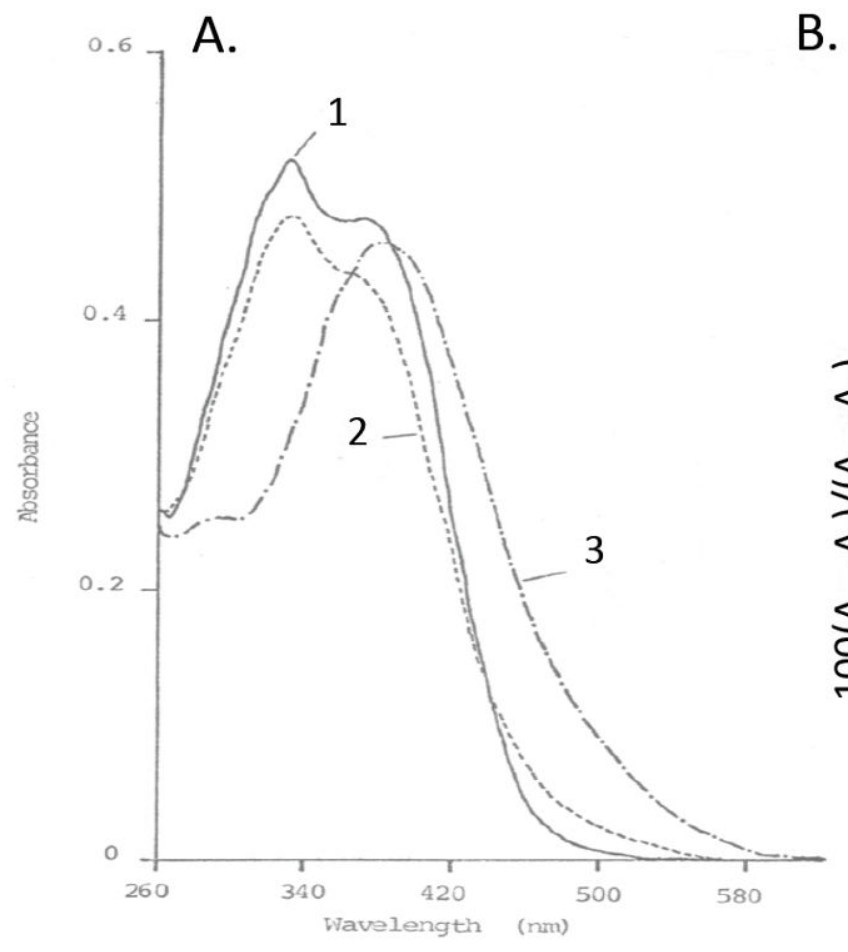

B.

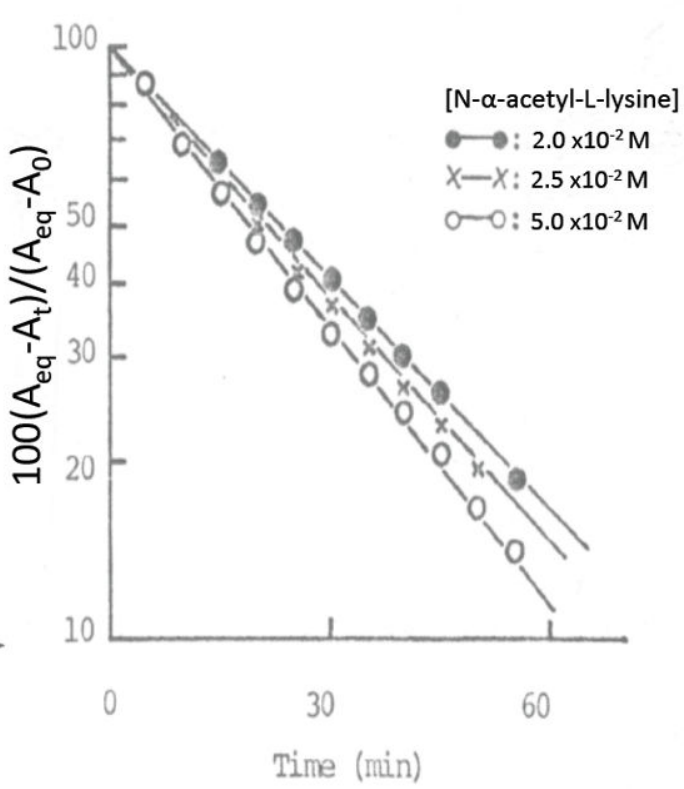

Fig. 5.

Determination of $k_{2}$ for the retinylidene Schiff base in a model system in a reaction between all-trans retinal and $\mathrm{N}$-a-acetyl-L-lysine in aqueous solution. A. Spectra of the components are illustrated. Curve 1, $13 \mu \mathrm{M}$ all-trans retinal in $78 \mathrm{mM}$ borate buffer $(\mathrm{pH} 8.2) / 22 \%$ ethanol in a total volume of $2.3 \mathrm{ml}$. Curve 2, $120 \mathrm{~min}$ after adding $0.2 \mathrm{ml}$ of $1 \mathrm{M} \mathrm{N}$-a-acetylL-lysine, the spectrum of the solution in equilibrium was measured. Curve 3, in order to protonate the retinylidene Schiff base a drop (ca $50 \mu \mathrm{l}$ ) of concentrated $\mathrm{HCl}$ was added and the spectrum was recorded. B. Dependence of the retinylidene Schiff base formation at three different concentrations of $\mathrm{N}$-a-acetyl-L-lysine all of which were excess over the concentration of all-trans retinal by the factor of larger than $10^{3}$. Under the conditions the reaction followed pseudo-first order kinetics. The retinylidene Schiff base formation was measured by monitoring the absorbance increase at $500 \mathrm{~nm}$, which is likely to involve formation of both of its unprotonated and protonated forms. The y-axis represents the percentage of remaining all-trans retinal after being converted to retinylidene Schiff base normalized to the equilibrium state; $y=100\left(A_{e q}-A_{t}\right) /\left(A_{e q}-A_{0}\right)$ where $A_{\text {eq }}$ represents the absorbance at $500 \mathrm{~nm}$ when the reaction became equilibrated, $A_{t}$ represents the absorbance at $500 \mathrm{~nm}$ at time $=\mathrm{t}$, and $\mathrm{A}_{0}$ represents the absorbance at $500 \mathrm{~nm}$ at $\mathrm{t}=0$. The pseudo-first order rate constant of retinylidene $\mathrm{N}$-alpha-acetyl-L-lysine formation $\left(\mathrm{k}_{\text {pseudo }}\right.$ ) (under the $\mathrm{N}$ alpha-acetyl-L-lysine excess) depended on the concentration of N-alpha-acetyl-L-lysine as shown in B. The regeneration rate of the model Schiff base $k_{2}$ was calculated from the tangent of the line when we plotted $\mathrm{k}_{\text {pseudo }}$ against [N-alpha-acetyl-L-lysine] (not shown). 
Table 1

The C6-C15 distance of bovine rhodopsin and intermediates calculated based on structural data by using PyMOL.

\begin{tabular}{lcccc}
\hline & Configuration & Property & PDB file & C6-C15 Distance in Å \\
\hline Rhodopsin & 11-cis & Stable & $1 \mathrm{U} 19$ & 10.0 \\
Isorhodopsin & 9-cis & Stable & 2PED & 9.9 \\
Bathorhodosin & all-trans & Transient & 2G87 & 10.3 \\
Metarhodopsin II & all-trans & Transient & 3PX0 & 11.0 \\
\hline
\end{tabular}

Photochem Photobiol Sci. Author manuscript; available in PMC 2015 October 30. 